\title{
INVESTIGACIONES
}

\section{TRANSFORMACIONES DEL PERFIL VALORICO EN ESTUDIANTES \\ DE LA CARRERA DE OBSTETRICIA Y PUERICULTURA DE LA FACULTAD DE MEDICINA DE LA UNIVERSIDAD DE LA FRONTERA DURANTE SU TRAYECTORIA ACADEMICA*}

Transformation of Value Profile on student of Obstetric and Puericulture in the Faculty of Medicin, Universidad de La Frontera, through his Academic Trajectory

\author{
Eliana Carrasco Delgado ${ }^{1}$, Sonia Osses Bustingorry ${ }^{2}$ \\ ${ }^{1}$ Universidad de La Frontera, Facultad de Medicina, Departamento de Ciencias Básicas, \\ Unidad de Bioquímica. Casilla 54-D, Temuco, Chile. lcarras@ufro.cl \\ ${ }^{2}$ Universidad de La Frontera, Facultad de Educación, Depto. de Educación, Casilla 54-D, Temuco, Chile. \\ sosses@ufro.cl
}

\section{Resumen}

Se presentan los resultados del análisis comparativo de datos obtenidos en la aplicación del instrumento "Value Survey" que mide el Perfil Valórico -adaptado por Brinkmann et al. (1999) para Chile- a veinte alumnos de la Carrera de Obstetricia y Puericultura al ingreso y al término de su formación profesional; ejercicio para el que se utilizó la prueba t de student para grupos relacionados.

Durante la trayectoria académica un $75 \%$ de los componentes de los Perfiles Valóricos reducen su importancia como elementos de influencia para el comportamiento y las decisiones que organizan la vida cotidiana de los alumnos.

Palabras clave: perfil valórico, educación valórica, modificación valórica, diferencias valóricas.

\begin{abstract}
We present comparative data obtained under the application of an instrument called "Value Survey", employed to detect valoric profile levels, adapted to Chle by Brinkman et al. (1999). This instrument was applied to 20 students of Obstetric and Care Child at the beginning and after their final examination to obtain their degree. The t-student test was employed to compare both groups.

We found through their academic endeavour al $75 \%$ decrease on their valoric profile principles as an element which influences their behaviour and decisions as they become professionals.
\end{abstract}

Key words: valoric profile, valoric education, valoric modification, valoric differences.

* $\quad$ Proyecto DIUFRO 120624, de la Dirección de Investigación y Desarrollo, Universidad de La Frontera. 


\section{INTRODUCCION}

1. Contextualización. La emancipación de la familia, la aguda conciencia de sí mismo, el volcarse hacia la realidad externa de los pares, el comienzo de un período de transición a la vida adulta y el ajuste a las nuevas demandas sociales son fenómenos asociados a la adopción de valores en el adolescente; en esos años se define la orientación que el individuo mantendrá en su vida respecto a metas, fines y proyectos. En este proceso, según Berkowitz (1998), los agentes educativos que contribuyen a configurar la forma de pensar y de actuar, especialmente en la juventud, son la familia, la escuela, el entorno social y los medios de comunicación; entre los últimos, la televisión es la que posee mayor influencia en la infancia, como agente de consumo y conformador de valores.

En lo referido a la experiencia universitaria, durante el transcurso de los años de estudio los alumnos, además de la progresiva independización de su familia de origen, han adquirido nuevas amistades y mayor libertad para elegirlas, han ido tomando decisiones propias, adquiriendo mayor madurez y responsabilidad; se han adscrito al espíritu de su Carrera, han recibido la influencia de sus compañeros, de sus profesores y de la Institución en general (Carrasco y Osses 2005).

Al dialogar con generaciones de estudiantes de Carreras del área de la salud se confirma que los valores adquiridos a lo largo de su vida son los que les han entregado su familia, su colegio y su entorno social. No obstante, es evidente que se precisan más estudios empíricos acerca de cómo se originan los valores, cómo operan en el ser humano y como éstos fluctúan a lo largo de su vida $-\mathrm{y}$ particularmente a través del proceso formativo universitario-, lo que significa construir variados instrumentos que puedan ser aplicados a la mayor cantidad posible de personas, lo que permitiría obtener conclusiones de mayor validez (Carrasco y Osses 2005). Es así que el presente trabajo pretende hacer un análisis descriptivo de cómo fluctúa el Perfil Valórico de los alumnos de ambos sexos que han optado por la Carrera de Obstestricia y Puericultura, desde el ingreso a la Carrera hasta la etapa de finalización de ésta. ¿Cómo se comporta el perfil valórico de los alumnos en el proceso de formación académica? es la pregunta que orienta esta investigación.

2. Valores y Educación. El crecimiento del ser humano, su perfeccionamiento, el cultivo de sus facultades, la búsqueda de la realización personal y social suponen considerar los valores, de modo que educar significa descubrir, incorporar y configurar valores, y educarse es sinónimo de internalizar valores para organizar la vida según ellos, actuando posteriormente de acuerdo a ellos. En este sentido, la formación de valores en educación es el medio para contextualizar y hacer ontológicamente significativos los contenidos y aprendizajes asociados a ellos. En este ámbito se diferencian proyectos educativos que en términos de contenidos o áreas de conocimiento apuntan a los mismos aspectos; de lo que se haga en este ámbito depende la diferenciación y posicionamiento de cada proyecto educativo; este es el ámbito en el que la Carrera de Obstetricia y Puericultura de la Facultad de Medicina de la Universidad de La Frontera puede diferenciarse tanto de otras Carreras del área biomédica de la misma facultad como de las mismas Carreras de otras casas de estudios.

En este sentido, la formación valórica -además de facilitar el aprendizaje contextualizado y significativo de los alumnos y de lograr identidad o diferenciación respecto 
de otras Carreras, potenciando su competitividad- también representa el nivel en el que se asume la responsabilidad social de cada proyecto educativo, al vincularlo con determinados proyectos sociales compatibles con los lineamientos valóricos optados en cada proyecto educativo.

Los valores son fines o metas de la educación y verdaderos criterios de evaluación de la calidad de la educación. Así, tanto mejor sea la educación, más alta y exigente será la jerarquía de valores que la sustenta. Según Moratinos (1985), los valores dotan al hecho educativo de una meta o marco de actuación amplio y el educador debe programar o planificar su labor docente apoyándose en el máximo nivel de abstracción, que es el axiológico o de los valores, en estrecha conexión con su meta. En esta dirección es que las distintas facultades y Carreras de la Universidad de La Frontera han iniciado un proceso de innovación docente orientado a mejorar la calidad educativa de los currículos que descansa en parte importante en el desarrollo y fortalecimiento de la formación valórica.

Durante los ciclos formativos los estudiantes de Obstetricia y Puericultura, así como todos los alumnos del área de la Salud, se relacionan con profesionales que a través del ejemplo van practicando con ellos valores por lo que se considera importante hacer un adecuado seguimiento en la estimación valórica de los estudiantes universitarios, y por ende de su perfil valórico, para perfeccionar la formación ética de los estudiantes. Los resultados de tales estudios pueden constituirse en indicadores útiles para apreciar el grado hasta el cual la Carrera está alcanzando sus objetivos: formar profesionales con un alto sentido del deber, altruista, profesional, capaz de reconocer sus limitaciones, íntegro, honesto, respetuoso de los otros y no discriminador. Por esta razón el investigar en torno a cómo fluctúa el perfil valórico de los alumnos de la Carrera de Obstetricia y Puericultura a lo largo de su trayectoria académica sería un aporte significativo para la comunidad universitaria, pues podría ayudar a promover cambios en la Misión y Visión de las Instituciones y Carreras, en las mallas curriculares, etc. Un análisis de este tipo reflejaría cómo está cumpliendo su Misión la institución y, lo que es más importante, si los docentes están contribuyendo en la mantención, fortalecimiento, mejoría y acrecentamiento de los valores iniciales de nuestros alumnos; que como profesionales que estarán inmersos en la modernización y el uso de nuevas tecnologías, se enfrentarán a realidades como la privatización de la salud y la globalización a todo nivel, será entonces cuando su riqueza valórica les proporcione la fortaleza necesaria para abordar tales desafíos.

La forma en que se asume este desafío de mejoramiento en la actualidad, dentro de un modelo curricular flexible, es incluyendo un conjunto de contenidos culturales básicos relacionados con el momento presente, relevantes para toda edad y de gran trascendencia para la juventud; son temáticas emergentes que se refieren a categorías valóricas y se conocen como Temas Transversales. No obstante, según Chico, la educación de los valores no puede constituir una asignatura dentro de un horario determinado, sino que es un proceso más profundo que se construye en torno a valores permanentes como la libertad y la felicidad. Es un proceso de naturaleza cultural, es decir, debe ser un proceso transversal, permanente y central en la formulación de proyectos educativos y diseño de currículos educativos. Fernández (1999) plantea que si se pretende lograr la educación integral de la persona para formar ciudadanos autónomos, conscientes, participativos y críticos, los temas transversales desempeñan un rol relevante y dan 
sentido al aprendizaje de contenidos, de manera que sean éstos los que fundamenten la adquisición de valores.

Los temas transversales poseen componentes valorativos y actitudinales, lo que contribuye al desarrollo ético-valórico de la persona; aspiran a que los alumnos se sensibilicen con las situaciones que enfrenten, adquiriendo actitudes positivas y comportamientos coherentes con sus principios personales, contribuyendo de ese modo a fomentar una postura ética que permita mejorar la convivencia de la sociedad en el futuro (Martínez 1995).

En el plano de la formación ética los temas transversales propician el desarrollo de capacidades y aprendizajes: autoconocimiento, autoestima y valoración positiva de la vida; pensamiento moral autónomo; capacidad de autorregular la conducta; reconocimiento y adhesión a los valores universales de la dignidad humana; capacidad de diálogo y participación democrática; compromiso crítico con la realidad social; comprensión crítica y respeto por las normas de convivencia.

3. Etica, Valores y complejización de la sociedad. Marlasca (1997) señala que se puede entender la ética como una disciplina que enseña cuál debe ser el comportamiento correcto del ser humano, entendiéndole como la moral vivida, ejercida y practicada, que comprendería un conjunto de reglas, normas, mandatos, tabúes y prohibiciones que regulan y guían la conducta y el comportamiento humano. Así entendida, la ética sería la búsqueda racional de rectitud en lo cotidiano, el empeño que pone cada persona para dar sentido a su propia libertad; su tema de fondo es la relación entre libertad y responsabilidad. La ética hace referencia al deber ser y deber hacer del ser humano, que por ser reflexivo es capaz de darse cuenta de sus actos, juzgarlos, valorarlos y orientarlos hacia metas que permitan lograr la felicidad (Magendzo 1997).

En el ámbito de la biomedicina y las profesiones médicas los valores éticos se han ido asociando progresivamente a las discusiones sobre los conflictos generados a partir del manejo técnico de la vida y del medio ambiente que la sustenta, dando origen a un campo interdisciplinario, denominado bioética. Surge en la segunda mitad del siglo XX, correspondiendo a una ética aplicada; es una reflexión interdisciplinaria tendiente a la resolución de los conflictos derivados de la manipulación técnica de la vida y del medio ambiente que le sustenta, que puedan representar una amenaza para la vida individual y colectiva. Hace de la naturaleza y de la vida, en su conjunto, objetos de interés moral ${ }^{1}$. Por lo tanto, la bioética vela por las relaciones del hombre con la naturaleza y con las demás personas, ya sean contemporáneos o para las futuras generaciones.

Por otro lado, los profundos cambios producidos por los fenómenos de integración territorial y globalización han relevado, hechos visibles, personas que pertenecen a distintas tradiciones o formas socioculturales, las cuales demandan condiciones de igualdad, justicia, libertad, solidaridad, equidad en la administración de recursos para la salud, construyendo una nueva ética pública en el marco de la globalización, de la mano de la bioética.

Los profesionales del área de la Salud -y los alumnos de Obstetricia y Puericultura que están finalizando sus estudios universitarios- se incorporarán paulatinamente a la

1 Escribar, A. "Bioética, su Sentido y su Función". Revista Médica. Vol. 14 № 2, abril 2003. www. clinicalascondes.cl/areaacademica/revista_medica_abril_2003/articulo_006html 
citada contingencia, pudiendo realizar, de acuerdo a sus principios y valores y con un profundo respeto por la vida humana, una encomiable labor, comenzando por participar en los equipos multiprofesionales e interdisciplinarios que conforman los Comités de Etica en su lugar de trabajo o contribuyendo a que se formen si aún no existen ${ }^{2}$.

Por otra parte, en el ámbito de la salud la Medicina ha evolucionado hacia una tecnología de la salud, lo que ha aportado grandes beneficios contra la enfermedad y a favor del bienestar físico del ser humano; no obstante, esto ha despersonalizado y deshumanizado la práctica médica, la persona ha perdido su unidad como ser humano. Humanizar la salud es una necesidad imprescindible y se constituye en un reto para los profesionales de esta área, en la que se incluyen los egresados de las Carreras en cuestión; el escenario en el que corresponderá ejercer la práctica profesional se caracterizará por la privatización de una práctica tradicionalmente concebida como derecho ciudadano e implementación pública, de lo que es evidencia la reestructuración del Ministerio de Salud y la Reforma del Sistema Previsional Chileno ocurrida a comienzos de los 80 . Actualmente existen empresas privadas que ofrecen a los trabajadores atención médica, reconocida como más eficiente y eficaz, aunque con un excesivo fin de lucro, mientras que aún subsiste un sistema público, financieramente restringido, para los sectores más pobres de la población ${ }^{3}$.

\section{MARCO TEORICO}

1. Los valores. Los valores constituyen el eje central de la cultura, pues son un tipo de conocimiento social que funciona para facilitar la adaptación de cada individuo a su entorno; tienen un carácter estable, lo que no implica que sean estáticos o rígidos; de hecho, pueden estimular hacia el cambio. Sin embargo, los cambios en ellos son lentos ya que son adquiridos a través de la socialización, que es un proceso activo, complejo, gradual y prolongado, que dura toda la vida. Son un conjunto de creencias que guían la conducta y que permiten decidir entre diversas opciones, siendo aprehendidos mediante el proceso de socialización, en el cual cobran relevancia diversas instituciones sociales: la institución gubernamental, que es la principal fuente de influencia para las otras instituciones sociales y cuyas funciones relevantes son mantener el orden, lograr el consenso y maximizar la producción económica; la religión, como iglesia organizada o sectas religiosas; el grupo de pares; los medios de comunicación y, por supuesto, la familia y el sistema educativo.

Debido a eso, cada persona llega a ser, en cada etapa de su desarrollo, el resultado de los procesos de interacción con el ambiente físico y social (Horton 1978). Se nace con condicionamientos biológicos, psicológicos y sociales, pero desde ellos el ser humano es capaz de actuar libremente, de irse comprendiendo a sí mismo y de realizarse a través de proyectos propios en colaboración con las demás personas (Gil 1998).

2 San Miguel, G. (2002). "Medicina al Día”. Sociedad Médica de Cautín. Revista Médica del Sur. 24 (2): 97-107.

3 Revista Saludy Futuro. "Debates de Coyuntura”. Una Visión Pluralista. www.saludyfuturo.cl/pags/deb_2002/ enero/cont_deb3,html 
Según lo señala Bunes (1997), la socialización es un proceso de transmisión cultural y transmitir los aspectos normativos de la cultura es equivalente a transmitir valores. Los padres, los profesores, los amigos, etc., constituyen modelos y en su comportamiento actualizan valores, los cuales son captados por los niños y las demás personas en general. Según Duarte (1985), considera la familia como una organización de personas que se influyen mutuamente, desempeñando distintos roles, sujetos a distintas normas y valores $\mathrm{y}$ cohesionados fundamentalmente por patrones afectivos.

2. Teoría de los valores de Schwartz y Bilsky. Desde el punto de vista educativo, constituye un problema saber cómo se originan los valores en el ser humano, cómo operan en él, cuál es su estructura psicológica y qué factores internos o externos influyen en su adquisición y/o modificación. Buscando una respuesta a tales interrogantes profesores pertenecientes a la Universidad Hebrea de Jerusalén, Israel y a la Universidad de Freiburg en Breisgau, República Federal de Alemania, se propusieron en 1987 investigar la existencia de una estructura universal de los valores humanos, formulando la teoría: "Estructura Psicológica de los Valores".

Schwartz y Bilsky (1987) definen VALOR como: el concepto que tiene un individuo de un objetivo (terminal-instrumental) transituacional, que expresa intereses (individuales-colectivos o ambos) concernientes a un dominio motivacional (placer, poder ...) y que es evaluado en un rango de importancia (muy importante-sin importancia) como principio rector de su vida (Binkman y Bizama 2002).

A partir de esta definición reconocen cinco facetas:

a) un concepto o creencia.

b) que atañe a situaciones finales o comportamientos deseables.

c) trasciende situaciones específicas.

d) guía, selecciona y evalúa comportamientos y eventos.

e) están ordenados entre sí por importancia relativa.

De acuerdo con Brinkman y Bizama (2002), en razón de lo anterior dichos autores postulan que el contenido escencial de un valor radica en el tipo de preocupación motivacional, propósito u objetivo que le da origen, y que se expresa en la actuación del sujeto. En atención a ello, propusieron una tipología de los diferentes contenidos de valores, al inferir, de algunos estudios previos (cf. Kluckhohn 1951; Parsons 1957; Rokeach 1973), que éstos representan, en la forma de objetivos conscientes, tres requisitos universales de la existencia humana que están presentes en todos los individuos en todas las sociedades:

a) necesidades de los individuos como organismos biológicos.

b) requisitos de interacción social coordinada.

c) necesidades de sobrevivencia y bienestar de los grupos.

De estos tres requisitos o necesidades humanas universales anteriormente señalados, Schwartz y Bilsky derivaron once tipos de motivaciones de valores. 
a) Autodirección. Orientación a ser independiente en la acción, el raciocinio y la afectividad, a ser autoafirmado como persona, con confianza en sí mismo. Se retroalimenta a partir de una búsqueda constante retroalimentación que confirme la percepción de autoeficacia, la que produce grados de compensación que confirman las propias capacidades de decisión y acción.

b) Estímulo. Relacionado con los requerimientos de valores de autodirección (Schwartz y Bilsky 1990), su eje es el "apetito" emotivo, la novedad y el desafío en la vida (una vida variada, excitada, osada, curiosa).

c) Hedonismo. Determinado por las necesidades orgánicas del sujeto y del placer asociado a la satisfacción de ellas. Se instaura simplemente por la obtención de placer o gratificación sensual para el sujeto mismo (placer, disfrutar la vida) (saludable).

d) Logro (Realización). Se define por la búsqueda del éxito personal, demostrándose competente para cumplir con los estándares sociales. Según Schwartz y Bilsky (en Brinkman y Bizama 2002) para que los individuos lleguen a obtener recursos de supervivencia, deben alcanzar como requisito previo una realización competente, a objeto de que la interacción social y el funcionamiento institucional sean exitosos.

e) Poder. Orientación a alcanzar un estatus de logro y de prestigio social, de control o dominio de las personas y de los recursos (autoridad, riqueza, poder social, conservación de la imagen pública, reconocimiento social, ser influyente).

f) Seguridad. Búsqueda de realización en la armonía y estabilidad de la sociedad, de sus relaciones y de sí mismo, como una proyección de las necesidades de los organismos de sobrevivir y evitar las amenazas a su integridad.

g) Conformidad. Orientación a restringir acciones, inclinaciones y probables impulsos que pueden alterar o dañar a otros y violar expectativas de normas sociales.

h) Tradición. Grado de respeto, dedicación y aceptación de las costumbres e ideas que la propia cultura o religión imponen sobre uno mismo (respeto por la tradición, humilde, devoto, aceptar mi parte en la vida, moderado, respeto a los padres y mayores).

i) Espiritualidad. Búsqueda de una armonía interior a través de la trascendencia de la realidad diaria.

j) Benevolencia. Preocupación por el bienestar del prójimo a través de la interacción diaria de los individuos, necesidad de una interacción positiva entre los sujetos para promover el florecimiento de grupos y la necesidad orgánica de afiliación. Orientación a la preservación y mejora del bienestar de la gente con la cual está el sujeto en frecuente contacto personal (servicial, leal, indulgente, honesto, responsable, amistad verdadera, amor maduro).

k) Universalismo. Aprecio del entendimiento, la tolerancia y la protección. Su propósito es lograr el bienestar de todas las personas y de la naturaleza en general.

Para verificar la universalidad de la estructura y relación dinámica entre los valores, Schwartz y Bilsky modificaron el "Value Survey" utilizado originalmente por Rokeach (1973), de modo que los sujetos asignaran a cada uno de los valores incluidos (30 terminales y 26 instrumentales) el grado de importancia que consideraban que tenía para ellos como "principio guía de su vida"; con este instrumento realizaron investigaciones transculturales. En este marco -el de verificar la universalidad de la estructura psicológica de valores y luego de efectuar en 1991 la adaptación para Chile del "Cues- 
tionario de Valores"- Brinkmann realizó en la ciudad de Concepción una investigación sobre la influencia de los estudios universitarios en el perfil valórico de los jóvenes, comparando dos universidades con diferencias sustantivas en el planteamiento de su Proyecto Educativo: Universidad Católica de la Santísima Concepción, con orientación religiosa, y Universidad de Concepción, con orientación laica. Considerando los respectivos Proyectos Educativos, elaboró un Perfil Valórico Ideal para los estudiantes, que posteriormente comparó con el Perfil Valórico Real mostrado por los alumnos en sus respuestas al Cuestionario de Valores. Utilizando la misma teoría, Sáiz, en la Región de La Araucanía, observó los valores de estudiantes universitarios mapuches y los comparó con valores de alumnos no indígenas y de alumnos mapuches que en 12 años de la educación formal se han asimilado al grupo cultural dominante. Concluye que alumnos identificados con su cultura mapuche originaria estiman más importante la conservación del statu quo, valoran en forma global el bienestar de la humanidad y la protección de la naturaleza, respetan las prácticas tradicionales y religiosas del grupo, tienen mayor grado de conformidad o autorrestricción frente a las expectativas y normas sociales, dan mayor valor a la seguridad, que es la protección de la estabilidad y armonía personal y social. Los estudiantes no mapuches y aquellos indígenas adscritos a la cultura chilena dominante, en tanto, privilegian valores relativos a la autopromoción, como el logro personal, el dominio sobre las personas o los recursos y el hedonismo o gratificación sensual (Brinkman y Bizama 2000).

Durante 2002, en la Universidad de La Frontera, las profesoras Carrasco y Osses (2005) investigaron el Perfil Valórico de alumnos que ingresaron a la Carrera de Obstetricia y Puericultura en un esfuerzo por consolidar información para innovar en el proyecto educativo de la Carrera al nivel de objetivos transversales del currículo relativos a la formación valórica. Concluyen de modo general que:

- El perfil valórico de estudiantes que tienen pertenencia a una formación común presenta características similares entre sí. Son altamente solidarios, valoran a su familia y a sus pares y en ellos la religión es importante como medio para buscar sentido a su existencia.

- Dentro del perfil valórico de los estudiantes se observa un orden o priorización de los valores sobre la base de una jerarquización de las motivaciones. La priorización va desde el mejor evaluado, que corresponde al valor "Seguridad Familiar", hasta el más rechazado o con menor evaluación, que corresponde al valor "Poder Social". Asimismo, la jerarquización en los dominios motivacionales considera la más alta evaluación para el dominio "Benevolencia", que integra los valores "sentido de pertenencia", "amor maduro", "amistad verdadera", "leal", "que ayuda", "honesto", "confiable" y "no rencoroso"; cuando, por otro lado, considera la más baja evaluación para el dominio "Poder", que comprende los valores "poder social", "riqueza", "reconocimiento social", "autoridad" y "conservar mi imagen pública".

3. Hipótesis o supuestos. El Perfil Valórico de los alumnos de la Carrera de Obstetricia y Puericultura se transforma durante la trayectoria académica en la dirección de asentar un alto sentido del deber, altruismo, profesionalismo, capacidad para reconocer sus limitaciones, integridad, honestidad, respeto por los otros y rechazo de la discriminación. 


\section{METODOLOGIA / METODO}

\section{Objetivos}

- Realizar un estudio descriptivo del perfil valórico en estudiantes de la Carrera de Obstetricia y Puericultura.

- Comparar el perfil valórico de los alumnos de la Carrera de Obstetricia y Puericultura al inicio y al final del proceso de formación profesional.

\section{Diseño}

En el marco de un estudio de tipo Descriptivo, se dispuso un Diseño Experimental del tipo preexperimento -con preprueba y postprueba en un solo grupo-, ya que no existía posibilidad de control de aspectos determinantes de la Trayectoria Académica ni se contaba con acceso a grupos de control como para el uso de diseños propiamente experimentales. No obstante estas restricciones de validez desde la perspectiva de un diseño Experimental Puro, cabe señalar la mantención de la misma muestra dentro del mismo universo en términos de sujetos en ambas mediciones. Esta cualidad valida la elección del diseño preexperimental indicado por sobre los de tipo Cuasiexperimentales aun cuando no dispone grupo control y las muestras provienen de grupos naturales.

\section{Sujetos}

El universo de referencia son los alumnos de Primer Nivel de la Carrera de Obstetricia y Puericultura, ingreso 2002, con un número de 30 alumnos, de ambos sexos, con una edad promedio de 18 años. De este universo se constituyó una muestra de 20 sujetos, los mismos referidos para las mediciones de 2002 y 2006.

\section{Instrumento}

Se utilizó el "Cuestionario de Valores", adaptación para Chile del "Value Survey" de Schwartz y Bilsky, realizada por Brinkmann et al. en 1991. Este instrumento incluye 56 valores específicos, los cuales son presentados en dos listados, los primeros 30 como Valores Meta o Terminales (constituyen estados finales deseables) y los restantes 26 como Valores Instrumentales (constituyen modos de conducta deseables), cada uno seguido de una frase explicativa.

Los valores que forman parte de los diferentes tipos están entremezclados a través del cuestionario. Los sujetos deben evaluar la importancia que tiene cada valor como principio que guía su vida, usando para ello una escala tipo Likert de nueve puntos, desde 1 hasta 7 .

Al final, el Cuestionario solicita datos sobre sexo, año de nacimiento y número de años en la universidad. 


\section{Plan de análisis de datos}

Se analizaron las respuestas al "Cuestionario de Valores" de Schwartz y Bilsky, adaptado, mediante el software computacional SPSS 10.0, obteniéndose estadística descriptiva (porcentajes, medidas de tendencia central, medidas de dispersión) para el grupo, en ambas mediciones y realizándose estimación estadística de diferencia de grupos a través de prueba t de student, en este caso para grupos relacionados.

\section{RESULTADOS}

La observación de los Perfiles Valóricos en los alumnos durante la trayectoria universitaria -transcurso desde el ingreso a la Carrera hasta su egreso- indica que el $100 \%$ de los Dominios o Dimensiones Motivacionales en las que se organiza el pool de valores que constituyen el perfil disminuyen su importancia para los alumnos como parámetros normativos que guíen la conducta y vida personal (ver gráfico 1). Así mismo ocurre cuando se observa el comportamiento directo de los valores, nivel en el que se reconoce que un $75 \%$ del total reduce su importancia como elementos de influencia para el comportamiento y las decisiones que organizan la rutina cotidiana de los estudiantes (ver gráfico 2).

\section{Gráfico 1}

Perfiles valóricos de los alumnos durante su trayectoria en la Carrera de Obstetricia y Puericultura

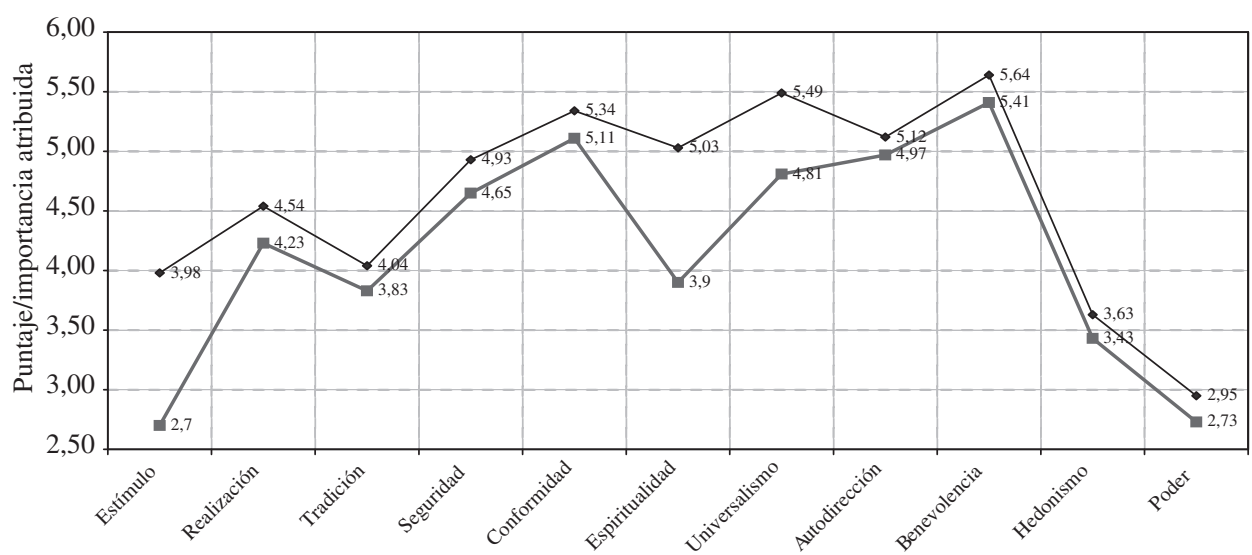

Dimensiones Motivacionales en las que se agrupan los valores

$\rightarrow 1^{\mathrm{a}}$ Medición $\rightarrow-2^{\mathrm{a}}$ Medición

No obstante, sólo algunos de esos cambios resultan estadísticamente significativos. En la tabla 1 se presenta un resumen del tipo y grado de fluctuación en los valores 
constitutivos del Perfil Valórico observado en la trayectoria académica de los alumnos de la Carrera de Obstetricia y Puericultura. Un detalle de la estadística descriptiva.

\section{Gráfico 2}

Perfil Valórico de los Alumnos durante su trayectoria en la Carrera de Obstetricia y Puericultura

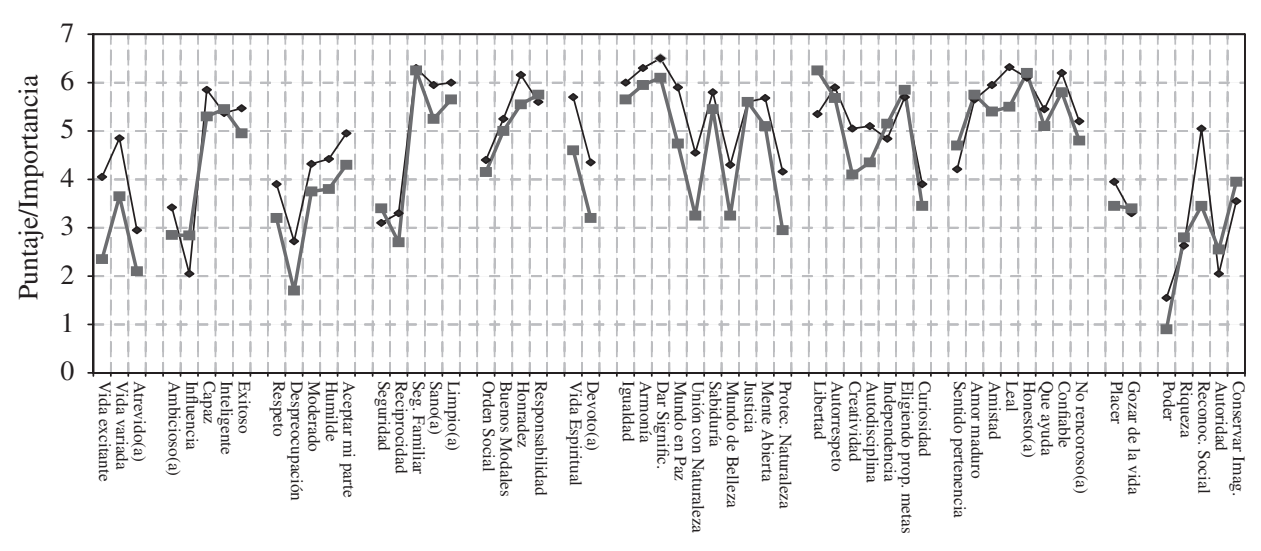

Valores

$\rightarrow 1^{\mathrm{a}}$ Medición $\rightarrow-2^{\mathrm{a}}$ Medición

\section{Tabla 1}

Comportamiento de los Dominios y Valores del Perfil Valórico de Alumnos (20) de Obstetricia y Puericultura entre su ingreso a la Carrera y su formación final

\begin{tabular}{|c|c|c|c|}
\hline \multirow{2}{*}{ Dominios e ítems } & \multicolumn{3}{|c|}{$\begin{array}{l}\text { Comportamiento observado en la } \\
\text { comparación antes / después }\end{array}$} \\
\hline & $\begin{array}{c}\text { sube, baja o se mantiene } \\
\text { la valoración* }\end{array}$ & $\begin{array}{l}\text { Media } 1^{a} \\
\text { medición }\end{array}$ & $\begin{array}{l}\text { Media } 2^{a} \\
\text { medición }\end{array}$ \\
\hline Dominio Universalismo & baja $* *(\mathbf{p}=0,023)$ & 5,49 & 4,81 \\
\hline Igualdad & baja & 6,00 & 5,65 \\
\hline Armonía Interna & baja & 6,30 & 5,95 \\
\hline Dar Significado a Mi Vida & baja & 6,50 & 6,10 \\
\hline Un Mundo en Paz & baja $^{* * *}(\mathbf{p}=0,001)$ & 5,90 & 4,75 \\
\hline Unión con la Naturaleza & baja $^{* *}(\mathbf{p}=0,028)$ & 4,55 & 3,25 \\
\hline Sabiduría & baja & 5,80 & 5,45 \\
\hline Mundo de Belleza & baja* $(\mathrm{p}=0,085)$ & 4,30 & 3,25 \\
\hline Justicia Social & estable & 5,60 & 5,60 \\
\hline
\end{tabular}




\begin{tabular}{|c|c|c|c|}
\hline \multirow{2}{*}{ Dominios e ítems } & \multicolumn{3}{|c|}{$\begin{array}{l}\text { Comportamiento observado en la } \\
\text { comparación antes / después }\end{array}$} \\
\hline & $\begin{array}{c}\text { sube, baja o se mantiene } \\
\text { la valoración* }\end{array}$ & $\begin{array}{l}\text { Media } 1^{a} \\
\text { medición }\end{array}$ & $\begin{array}{l}\text { Media } 2^{a} \\
\text { medición }\end{array}$ \\
\hline Mente Abierta & baja & 5,68 & 5,10 \\
\hline $\begin{array}{l}\text { Protector del Medio } \\
\text { Ambiente }\end{array}$ & baja* $(\mathrm{p}=0,083)$ & 4,16 & 2,95 \\
\hline Dominio Poder & baja $^{* *}(\mathbf{p}=\mathbf{0 , 0 1})$ & 2,95 & 2,73 \\
\hline Poder Social & baja & 1,55 & 0,90 \\
\hline Riqueza & sube & 2,63 & 2,80 \\
\hline Reconocimiento Social & baja $^{* *}(\mathbf{p}=0,021)$ & 5,05 & 3,45 \\
\hline Autoridad & sube & 2,05 & 2,55 \\
\hline Conservar Imagen Pública & sube & 3,55 & 3,95 \\
\hline Dominio Hedonismo & baja & 3,63 & 3,43 \\
\hline Placer & baja & 3,95 & 3,45 \\
\hline Gozar de la Vida & estable & 3,30 & 3,40 \\
\hline Dominio Autodirección & sube & 4,71 & 4,97 \\
\hline Libertad & sube $^{*}(\mathrm{p}=0,055)$ & 5,35 & 6,25 \\
\hline Autorrespeto & baja & 5,90 & 5,68 \\
\hline Creatividad & baja $^{* *}(\mathbf{p}=0,024)$ & 5,05 & 4,10 \\
\hline Autodisciplina & baja $^{* *}(\mathbf{p}=0,040)$ & 5,10 & 4,35 \\
\hline Independiente & sube & 4,84 & 5,15 \\
\hline Eligiendo mis Propias Metas & estable & 5,70 & 5,85 \\
\hline Curioso & baja & 3,90 & 3,45 \\
\hline Dominio Espiritualidad & Baja $^{* *}(\mathbf{p}=\mathbf{0 , 0 2 4})$ & 5,03 & 3,90 \\
\hline Vida Espiritual & baja** $(\mathbf{p}=\mathbf{0 , 0 2 5})$ & 5,70 & 4,60 \\
\hline Devoto & baja* $(\mathrm{p}=0,087)$ & 4,35 & 3,20 \\
\hline Dominio Benevolencia & baja & 5,64 & 5,41 \\
\hline Sentido de pertenencia & sube & 4,21 & 4,70 \\
\hline Amor Maduro & estable & 5,65 & 5,75 \\
\hline Amistad Verdadera & baja* $(\mathrm{p}=0,069)$ & 5,95 & 5,40 \\
\hline Leal & baja $^{* *}(\mathbf{p}=0,014)$ & 6,32 & $\mathbf{5 , 5 0}$ \\
\hline Honesto & estable & 6,10 & 6,20 \\
\hline Que Ayuda & baja & 5,45 & 5,10 \\
\hline Confiable & baja* $(\mathrm{p}=0,072)$ & 6,20 & 5,80 \\
\hline No Rencoroso & baja & 5,20 & 4,80 \\
\hline
\end{tabular}




\begin{tabular}{|c|c|c|c|}
\hline \multirow{2}{*}{ Dominios e ítems } & \multicolumn{3}{|c|}{$\begin{array}{l}\text { Comportamiento observado en la } \\
\text { comparación antes / después }\end{array}$} \\
\hline & $\begin{array}{l}\text { sube, baja o se mantiene } \\
\text { la valoración* }\end{array}$ & $\begin{array}{l}\text { Media } 1^{a} \\
\text { medición }\end{array}$ & $\begin{array}{l}\text { Media } 2^{a} \\
\text { medición }\end{array}$ \\
\hline Dominio Estímulo & baja $* *(\mathbf{p}=\mathbf{0 , 0 2 0})$ & 3,98 & 2,7 \\
\hline Vida Excitante & baja $* *(\mathbf{p}=0,009)$ & 4,05 & 2,35 \\
\hline Vida Variada & baja** $(\mathbf{p}=\mathbf{0 , 0 3 6})$ & 4,85 & 3,65 \\
\hline Atrevido & baja & 2,95 & 2,1 \\
\hline Dominio Realización & baja & 4,51 & 4,38 \\
\hline Ambicioso & baja & 3,42 & 2,85 \\
\hline Influyente & sube & 2,05 & 2,84 \\
\hline Capaz & baja* $(\mathrm{p}=0,053)$ & 5,85 & 5,30 \\
\hline Inteligente & estable & 5,37 & 5,45 \\
\hline Exitoso & baja & 5,47 & 4,95 \\
\hline Dominio Tradición & baja $^{* *}(\mathbf{p}=\mathbf{0 , 0 3 4})$ & 4,04 & 3,83 \\
\hline Respeto & baja & 3,9 & 3,2 \\
\hline Despreocupación & baja & 2,72 & 1,7 \\
\hline Moderado & baja & 4,32 & 3,75 \\
\hline Humilde & baja & 4,42 & 3,8 \\
\hline Aceptar mi parte en la vida & baja & 4,95 & 4,3 \\
\hline Dominio Seguridad & baja & 4,93 & 4,65 \\
\hline Seguridad & sube & 3,10 & 3,40 \\
\hline Reciprocidad de favores & baja & 3,30 & 2,70 \\
\hline Seguridad familiar & estable & 6,30 & 6,25 \\
\hline Sano & baja & 5,95 & 5,25 \\
\hline Limpio & baja & 6,00 & 5,65 \\
\hline Dominio Conformidad & baja & 5,34 & 5,11 \\
\hline Orden social & baja & 4,40 & 4,15 \\
\hline Buenos modales & baja & 5,25 & 5,00 \\
\hline $\begin{array}{l}\text { Honrar a los padres y } \\
\text { mayores }\end{array}$ & baja* $(p=0,093)$ & 6,16 & 5,55 \\
\hline Responsable & baja & 5,60 & 5,75 \\
\hline
\end{tabular}

* En negro y con dos estrellas se indican las diferencias que resultan estadísticamente significativas $(\mathrm{p}=0,05)$. Se han indicado también, con una estrella, aquellas diferencias que sin ser estadísticamente significativas, por su cercanía con el límite de probabilidad de error aceptable, resultan destacables respecto del conjunto. 
En el nivel de los dominios motivacionales que organizan el Perfil Valórico, aquellos que observan bajas estadísticamente significativas son Universalismo, Espiritualidad, Tradición, Poder y Estímulo.

El descenso de la importancia de los valores orientados motivacionalmente por el Universalismo representa una disminución de la necesidad de los alumnos por relaciones basadas en el entendimiento, la apreciación, la tolerancia y la protección mutua. Se reduce el interés por lograr el bienestar de todas las personas y de la naturaleza en general. Estas condiciones o exigencias pierden influencia en la organización de la conducta social cotidiana de los alumnos. Así mismo ocurre con los valores orientados motivacionalmente por el dominio Tradición; disminuyen su influencia en la conducta de los alumnos el respeto, la dedicación y la aceptación de las costumbres e ideas que la propia cultura o religión imponen sobre uno mismo. Cualidades como humildad, ser devoto, moderado, respetar a los padres y mayores pierden atractivo o interés.

La modificación de estos dominios en el Perfil Valórico resulta consistente con la disminución del interés por dotar a la vida de un significado y coherencia frente al aparente sinsentido de la existencia diaria (Buber 1958, Niebuhr 1956, Yinger 1957) y buscar la armonía interior a través de la trascendencia de la realidad diaria, representada en la disminución de la apreciación de los valores integrados en el dominio motivacional Espiritualidad.

Acompaña esta modificación del perfil valórico la reducción del interés por alcanzar un estatus de logro y de prestigio social, haciéndose especialmente irrelevante en la jerarquía de valores de los alumnos la búsqueda de reconocimiento social y el comportamiento de adaptación social subsecuente. Esto resulta consistente en el escenario de disminución de la valoración, motivación o interés por una vida espiritual, guiada por motivos trascendentes, o por valores tradicionales impuestos por la cultura o la religión. Sin estos parámetros de referencia se desdibuja el significado de los valores integrados en el dominio Poder.

Considerados estos Dominios Motivacionales, los valores en los que se observa una reducción significativa de su importancia o interés para los alumnos (ver gráfico 3) son relativos a la mantención de la paz en el mundo y el rechazo de guerras y conflictos; el respeto, la integración y defensa de la naturaleza; la búsqueda de belleza en la naturaleza y las artes. Esto en el dominio Universalismo. En el dominio motivacional Espiritualidad, los valores que más disminuyen su importancia o interés para los alumnos se refieren al mantenimiento de las creencias y fe religiosas y la consideración de aspectos espirituales, no materiales, en las decisiones o actuar cotidiano, a lo que se agrega una significativa disminución de la apreciación de valores relativos a la búsqueda de respeto y aprobación de los demás en el dominio motivacional Poder.

Finalmente, dentro del dominio motivacional Estímulo, los valores que observan una disminución significativa son relativos a la búsqueda de experiencias estimulantes, de cambio, desafiantes, novedosas. Adquiere valor la búsqueda de un estilo de vida estable, seguro, controlable.

No obstante los resultados obtenidos en el nivel anterior -el de los dominios motivacionales- resulta importante relevar una serie de otros valores que integran el perfil motivacional y que muestran rebajas estadísticamente significativas en su estimación de importancia o interés por los alumnos, aun cuando los Dominios Motivacionales a los que pertenecen no han experimentado una disminución de igual magnitud. El cambio 
en estos valores ayudan a ponderar las cualidades relevantes descritas en el cambio a nivel de Dominios.

\section{Gráfico 3}

Valores constitutivos de las Dimensiones del perfil valórico con disminución de importancia significativa

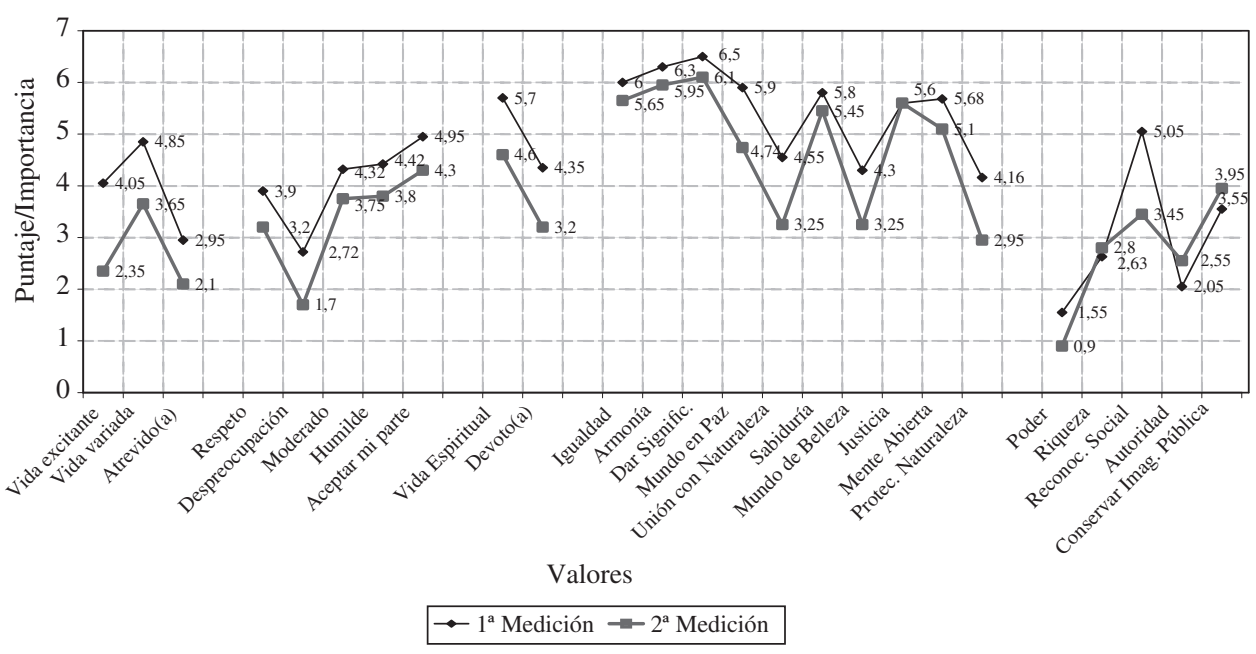

Es así que valores relativos a la originalidad y desarrollo de la imaginación, tanto como al fortalecimiento de la capacidad de autocontrol y resistencia a impulsos y tentaciones, experimentan las bajas de apreciación más significativas por parte de los alumnos, todos integrados en el dominio motivacional Autodirección. Otro valor que muestra una significativa reducción de su importancia para la organización de la rutina cotidiana de los alumnos es el relativo a la amistad, identificación y fidelidad grupal de cualquier naturaleza, en el ámbito motivacional Benevolencia, dentro del que se agregan -aun cuando sin un grado aceptable de significación- la disminución en la apreciación de valores relativos a la mantención de una conducta y actitud confiable, responsable, tanto como al desarrollo de relaciones cercanas de amistad y apoyo psicosocial.

Finalmente -como en el caso del valor anterior, aun cuando sin un grado aceptable de significación- cabe señalar la baja de apreciación observada en valores relativos al respeto de los padres y mayores en el dominio Conformidad, tanto como de valores relativos al desarrollo de una conducta social competente y eficaz; esto, en el dominio Realización.

Una mención especial amerita la referencia a los valores que -dentro de un perfil de disminución de la estimación general de los valores y dominios motivacionales en los Perfiles Valóricos- demostraron un incremento de su importancia o interés para los alumnos. Aun cuando el único valor que observa un alza estadísticamente significativa de apreciación o de incremento de su importancia para los alumnos es libertad de acción y pensamiento, en el dominio motivacional Autodirección, ámbito en el que se reconoce 
también un incremento de la importancia del valor de autonomía, autosuficiencia e independencia personal y psicosocial. Concomitante con estos incrementos se observa un aumento de la valoración de posesiones materiales y dinero, derecho a dirigir o mandar y a proteger la imagen personal; esto, en el ámbito Poder.

No obstante reconocerse una cierta orientación a la independización social y normativa en los cambios observados en los Perfiles Valóricos de los alumnos durante su trayectoria académica, destaca el hecho de observarse incremento en la apreciación de importancia o interés de los alumnos por valores relativos a la protección de "la patria" contra enemigos, en el dominio Seguridad, y a sentimientos de pertenencia o identificación con causas, grupos o personas; esto, en el ámbito Benevolencia.

\section{DISCUSION}

La comparación de los Perfiles Valóricos del grupo de alumnos de la Carrera de Obstetricia y Puericultura, obtenidos al inicio y término de su trayectoria académica, dan cuenta de la conformación de un esquema normativo, valórico y ético, fundado en el interés y bienestar personal, desvinculado de exigencias de respeto, preocupación y protección social. Se prescinde paulatinamente de las prescripciones conductuales y actitudes exigidas por la propia cultura, religión o institución de pertenencia. Se reconoce una orientación hacia la individualización y liberalización de la elección de objetivos e intereses vitales, disminución de la solidaridad y desarrollo de actitudes instrumentalizadoras de las relaciones en el ámbito personal y social tanto como natural, para las que la búsqueda de reconocimiento social no resulta funcional.

Esta conformación valórica se completa con un distanciamiento de la búsqueda de sentido trascendente a través de la religión y la fe, priorizándose un proceso de regulación de las emociones e impulsos mediante el desarrollo de la racionalización, intelectualización, en una lógica de naturaleza más bien científica que místico-religiosa. En este proceso de intelectualización se privilegia un estilo de funcionamiento social e intelectual formal, esquemático, propio de un estilo de funcionamiento cognitivo racional-científico, reduciéndose el interés por la actividad creativo-imaginativa en pos del fortalecimiento de los medios racionales, individuales e instrumentales, para la determinación de la conducta personal y social.

Esto que es observado a nivel de los dominios motivacionales en el comportamiento del Perfil Valórico de los alumnos de Obstetricia y Puericultura, se corresponde con lo observado en el nivel de los valores que integran estos dominios. Así es como -confirmando la orientación general hacia la independencia personal, el desarrollo de logros e intereses más bien individuales que colectivos y la liberalización de los marcos socionormativos- se reconoce una disminución de la importancia o interés por el desarrollo de relaciones de fraternidad, fidelidad, confianza y apoyo socioafectivo. Lo mismo ocurre con el valor del respeto a los padres, mayores o figuras de autoridad. Especialmente ilustrativo de esta orientación individualista en el desarrollo del Perfil Valórico de los alumnos de Obstetricia y Puericultura resulta la disminución de importancia para los alumnos de los estándares de desempeño socialmente establecidos, reemplazándose la orientación a la competencia/adaptación social por la de libertad de acción y pensamiento y la autonomía e independencia personal. 
En este marco, se reemplaza en forma progresiva durante la trayectoria académica la presencia de valores trascendentes, colectivos, solidarios e ideales por valores instrumentales vinculados al logro de posesiones materiales y dinero, poder y estatus social, económico y político.

No obstante lo anterior, se reconoce un incipiente incremento de valores vinculados a sentimientos patrióticos, relativos a la identidad nacional, tanto como a necesidades de construcción de identificaciones y sentimientos de pertenencia a causas o grupos de distinta naturaleza. Cabe señalar, no obstante, que -dado el desentendimiento progresivo de marcos de referencia normativos, y por lo tanto identitarios, de naturaleza sociocultural o macroinstitucional- estos procesos de integración o identificación personal se enfocan en instancias microsociales, intermediarias, que superan la referencia relativa a la familia pero que se distancian de identificaciones socioculturales generales, inclusivas, como estado, iglesia, nación, región, cultura, entre otras. Se propician condiciones para la identificación con la diferencia que se verifica en el nivel de organizaciones como grupos juveniles o tribus urbanas, sectas religiosas, gremios laborales, clubes temáticos (deportivos, culturales, artísticos, etc.), movimientos sociales, iniciativas políticas locales, etc.

Finalmente, el estudio deja planteada una serie de preguntas derivadas de la constatación de modificación del Perfil Valórico de los alumnos de Obstetricia y Puericultura durante su trayectoria académica: ¿estos cambios son propios del universo de alumnos constituido por la Carrera de Obstetricia y Puericultura o más bien son propios del proceso de formación universitaria en general?; por otro lado, ¿influye en estos cambios la experiencia educativa producida en los alumnos por los currículos educacionales que operacionalizan los distintos proyectos educativos o más bien responden a un proceso de maduración o desarrollo psicosocial propio de contextos como los que caracterizan la sociedad contemporánea posmoderna?

Para la resolución de estas preguntas se requiere la continuación de este estudio descriptivo en otros de naturaleza correlacional y explicativo, con diseños cuasiexperimentales, con mediciones antes/después y grupos de control de distinta naturaleza. Sin la resolución de las preguntas planteadas y la implementación de las investigaciones complementarias no es posible afirmar lo que los resultados refieren sobre la hipótesis de estudio, esto es su rechazo, por lo menos parcial, ya que las modificaciones del Perfil Valórico durante la Trayectoria Académica muestran un movimiento en el sentido contrario, o cuando menos inconsistente, con la dirección de asentar un alto sentido del deber, altruismo, profesionalismo, capacidad para reconocer sus limitaciones, integridad, honestidad, respeto por los otros y rechazo de la discriminación.

\section{BIBLIOGRAFIA}

Acevedo, A. et al. (1999). El proceso de la Entrevista. Conceptos y Modelos. México: Editorial Limusa.

Aguirre, A. (1995). Etnografía. Metodología Cualitativa en la Investigación Sociocultural. Barcelona: Editorial Boixareu Universitaria.

Berkowitz, M. (1998). Educación, valores y democracia. Madrid: Organización de Estados Iberoamericanos para la Educación, la Ciencia y la Cultura. 
Bertaux, D. (1976). El Enfoque Biográfico: su validez metodológica, sus potencialidades. Historias y Relatos de Vida: Investigación y Práctica en las Ciencias Sociales. México: SUR Ediciones.

Brinkmann, H. et al. (1993). Estudio de la Estructura Psicológica de los Valores Humanos. Informe final proyecto Fondecyt 90-0159, Dirección de Investigación, Universidad de Concepción, Chile.

Brinkmann, H. et al. (1999). Influencia de los Estudios Universitarios en el Perfil Valórico de los Jóvenes. Comparación de dos universidades. Memorias de la Quinta Conferencia Internacional de las Ciencias de la Educación. Camagüey, Cuba.

Brinkmann, H. et al. (2000). Estructura Psicológica de los Valores. Presentación de una Teoría (1). Sociedad Hoy, $\mathrm{N}^{\circ} 4$.

Buendía, L. et al. (1998). Métodos de Investigación en Psicopedagogía. Madrid: Editorial McGraw-Hill.

Bunes, M. et al. (1997). Educación y Desarrollo Humano: el papel de los valores desde el modelo Hall-Tonna. Educadores 182-183, año 39, vol. 40-41.

Carrasco, L y S. Osses (2007). Transformaciones del Perfil Valórico de Estudiantes de la Carrera de Obstetricia y Puericultura de la Facultad de Medicina de la Universidad de La Frontera durante su Trayectoria Académica: Resultados Preliminares. Actas VII Jornadas de Psicología Educacional. Universidad de La Frontera, Pucón.

Carrasco, L. y S. Osses (2005). Estudio del Perfil Valórico: el caso de los estudiantes de primer nivel de la Carrera de Obstetricia y Puericultura de la Facultad de Medicina de la Universidad de La Frontera, Temuco, Chile. Estudios Pedagógicos V XXXI, N 1: 7-32.

Carreras, Ll. et al. (1997). Cómo educar en valores. Materiales, textos, recursos y técnicas. Madrid: Ediciones Narcea S.A.

Carrillo, A. (1996). Los valores, el reto de hoy. Orientaciones para la implementación del proyecto de ética. Santafé de Bogotá: Cooperativa Editorial Magisterio.

Córdova, G (2007). Participación y Juventud. Taller de Capacitación, Innovación Educativa y Participación. Temuco, Chile.

Chico, P. (1993). Valores permanentes de la educación cristiana. Educadores $\mathrm{N}^{\circ}$ 122, Vol. XXV.

Denegri, M. y G. Martínez (2001). Proyectos de aula interdisciplinarios: Una mirada desde la psicología educacional. Psicología y Educación: Encuentros y Desencuentros. Departamento de Publicaciones. Universidad de La Serena, pp. 27-35.

Duarte, D. et al. (1985). Alcoholismo en Chile. Revista difusora de Prevención a la Salud 3 (1).

Fernández, J. (1999). Los valores en el marco de la educación primaria. Educadores $\mathrm{N}^{\circ}$ 189, Vol. 41.

Fierro, A. (1998). Relaciones Sociales en la Adolescencia. Desarrollo Psicológico en Educación. Palacios, J. (Comp). Psicología Evolutiva. Madrid: Editorial Alianza.

Gil, R. (1998). Valores humanos y desarrollo personal. Tutorías de educación secundaria y escuelas de padres. Madrid: Editorial Escuela Española S.A.

Hernández, R. et al. (1998). Metodología de la Investigación. México: Editorial McGraw-Hill.

Hopkins, D. (1989). Investigación en el aula. Guía del profesor. Barcelona: Promociones y Publicaciones Universitarias S.A.

Horton, P. et al. (1978). Sociología. México: Editorial McGraw-Hill.

Knust, R. (2007). Educación para la participación, una mirada europea. STOAS, Activating Knowledge. Villarrica, Chile.

Latorre, A. et al. (1996). Bases metodológicas de la investigación educativa. Barcelona: editor Jordi Hurtado.

Light, D. (1991). Sociología. Bogotá: Editorial McGraw-Hill.

Magendzo, A. et al. (1997). Los objetivos transversales de la educación. Santiago: Editorial Universitaria. 
Magendzo, A. et al. (1998). Los objetivos transversales en la reforma educativa chilena. Colección "el sembrador". Santiago: Editorial Universitaria.

Marlasca, A. (1997). Introducción a la Etica. San José: Editorial Universitaria Estatal a Distancia (EUNED).

Martínez, M. J. (1995). Los temas transversales. Argentina: Magisterio del Río de la Plata.

Medina, J. L. (1996). La credibilidad de la Investigación Científica Interpretativa. La Pedagogía del Cuidado: racionalidad, tradición y poder en el currículum de enfermería. Un estudio interpretativo. España: Universidad de Barcelona.

Mella, O. (2000). Grupos Focales. Técnica de Investigación Cualitativa. Santiago: Centro de Investigación y Desarrollo de la Educación (CIDE).

Ministerio de Educación (2005). Diagnóstico y recomendaciones en torno a la formación ciudadana. Revista de Educación: Pedagogía en Derechos Humanos 316: 32-37.

Ministerio de Educación (2005). Ley 19.979 consejos Escolares, "todos comprometidos por la Calidad de la Educación". Chile.

Moratinos, J. (1985). Aproximación al estudio filosófico de los valores y la educación. Educadores $\mathrm{N}^{\circ} 131$, Vol. XXVII.

Navarro, R. (2000). Metodología de la Investigación. Apuntes programa Magíster en Pedagogía y Gestión Universitaria. Santiago: Universidad Metropolitana de Ciencias de la Educación.

Oxman, C. (1998). La Entrevista de Investigación en Ciencias Sociales. Buenos Aires: Editorial Universitaria de Buenos Aires (EUDEBA).

Padilla, A. (1996). Educación en valores y su sentido. Pensamiento Educativo. Santiago.

Pérez, G. (1998). Investigación Cualitativa. Retos e Interrogantes. Tomo I: Métodos. Madrid: Editorial La Muralla S.A.

Pérez, G. (1998). Investigación Cualitativa. Retos e Interrogantes. Tomo II: Técnicas y análisis de datos. Madrid: Editorial La Muralla S.A.

RED PARTICIPA (2007). Municipio, Participación e Innovación Educativa. Resultados del proceso de diagnóstico Proyecto comunas de Temuco, Chol Chol, Imperial, Lautaro, Villarrica. Chile. www.redparticipa.cl

Rodríguez, G. et al. (1999). Metodología de la Investigación Cualitativa. Málaga: Ediciones Aljibe.

Salazar, J. M. (1984). Valores y motivaciones sociales. Psicología Social. México: Editorial Trillas.

Solar, M. T. (1999). Creatividad en educación. Concepción: Universidad de Concepción, Dirección de Docencia, Vicerrectoría Académica.

Stake, R. (1998). Investigación con estudio de casos. Madrid: Ediciones Morata.

Taylor, S. et al. (1996). Introducción a los métodos cualitativos de investigación. La búsqueda de significados. Barcelona: Editorial Paidós Ibérica S.A.

Tierno, B. (1996). Guía para educar en valores humanos (persona, familia, escuela, sociedad). Madrid: Taller de Editores S.A.

Usler, B. (1992). La educación de adultos como teoría práctica e investigación. Madrid: Editorial el Triángulo Cautivo, Morata.

Valenzuela, A. (1997). Persona y educación. Valparaíso: Ediciones Universitarias de Valparaíso. 
